\title{
Quasi-isometries between groups with infinitely many ends
}

Panos Papasoglu and Kevin Whyte

\begin{abstract}
Let $G, F$ be finitely generated groups with infinitely many ends and let $\pi_{1}(\Gamma, \mathcal{A})$, $\pi_{1}(\Delta, \mathcal{B})$ be graph of groups decompositions of $F, G$ such that all edge groups are finite and all vertex groups have at most one end. We show that $G, F$ are quasi-isometric if and only if every one-ended vertex group of $\pi_{1}(\Gamma, \mathcal{A})$ is quasi-isometric to some one-ended vertex group of $\pi_{1}(\Delta, \mathcal{B})$ and every one-ended vertex group of $\pi_{1}(\Delta, \mathcal{B})$ is quasi-isometric to some one-ended vertex group of $\pi_{1}(\Gamma, \mathcal{A})$. From our proof it also follows that if $G$ is any finitely generated group, of order at least three, the groups: $G * G, G * \mathbb{Z}, G * G * G$ and $G * \mathbb{Z} / 2 \mathbb{Z}$ are all quasi-isometric.
\end{abstract}

Mathematics Subject Classification (2000). 20F65, $20 \mathrm{E} 06$.

Keywords. Free products, quasi-isometries, ends of groups.

\section{Introduction}

One of the most appealing and influential theorems in geometric group theory is Stallings' Ends Theorem, [St1], [St2]. This theorem says that a finitely generated group splits as a free product or HNN-extension with finite amalgamation if and only if it has more than one end. The property of having infinitely many ends is geometric, in particular, it is invariant under quasi-isometry. One of the main goals of geometric group theory is to classify finitely generated groups up to quasi-isometry. Given that splitting over finite subgroups is invariant under quasi-isometry, it is natural to ask how the quasi-isometry type of a free product with finite amalgamation is related to the types of its factors.

This question is not as straightforward as one might think. It is not true that if $G$ and $G^{\prime}$ are quasi-isometric then $G * H$ and $G^{\prime} * H$ are. Examples of this can already be seen among finite groups: $\mathbb{Z} / 2 \mathbb{Z} * \mathbb{Z} / 2 \mathbb{Z}$ and $\mathbb{Z} / 2 \mathbb{Z} * \mathbb{Z} / 3 \mathbb{Z}$ are not quasi-isometric. Our first theorem shows that this is essentially the only source of examples.

Theorem 0.1. Let $A, B$ and $C$ be nontrivial groups. If $A$ and $B$ are quasiisometric then $A * C$ and $B * C$ are quasi-isometric unless $C$, and one of $A$ or $B$, are of order 2 . 
We note that some special cases of theorem 0.1 have been treated in $[\mathrm{P}]$, and $[\mathrm{W}]$.

Stallings' theorem gives splittings over finite subgroups, not free products. Our next theorem shows that from the quasi-isometric point of view, finite amalgamated products are free.

Theorem 0.2. Let $A$ and $B$ be groups, and $F$ a common finite proper subgroup. Unless $F$ is of index 2 in both $A$ and $B$ then $A * B$ and $A *_{F} B$ are quasi-isometric. Likewise, $A *_{F}$ and $A * \mathbb{Z}$ are quasi-isometric unless $F$ has index 1 in $A$.

From these theorems we get a complete classification of the quasi-isometry types of graphs of groups with finite edge groups:

Theorem 0.3. Let $G, H$ be finitely generated groups with infinitely many ends and let $\pi_{1}(\Gamma, \mathcal{A}), \pi_{1}(\Delta, \mathcal{B})$ be decompositions of $G, H$ in graphs of groups such that all edge groups are finite. If $\pi_{1}(\Gamma, \mathcal{A}), \pi_{1}(\Delta, \mathcal{B})$ have the same set of quasi-isometry types of vertex groups (without multiplicities) then $G$ and $H$ are quasi-isometric.

It is natural to ask for a converse. As it may be possible to split a vertex group, the naive converse cannot be true. By Stallings' theorem, a vertex group splits if and only if it has 2 or more ends. The obvious thing to do is to split until no more splitting is possible, in other words, until all vertex groups are finite or one ended. A finite graph of groups with this property is called terminal, and a group which has a terminal splitting is called accessible.

The Grushko-Neumann theorem shows that finitely generated, torsion-free groups, are accessible. It is also true that finitely presented groups are accessible $([\mathrm{D} 1])$. While it would be natural to think all finitely generated groups are accessible, indeed this was a conjecture for quite a while, it is not true ([D2]). It follows easily from the characterization of accessibility in [TW] that accessibility is a quasi-isometry invariant. Together with the earlier theorems, this yields:

Theorem 0.4. Let $G$ be an accessible group and let $\pi_{1}(\Gamma, \mathcal{A})$ be a terminal graph of groups decomposition of $G$. A group $G^{\prime}$ is quasi-isometric to $G$ if and only if it is also accessible and any terminal decomposition of $G^{\prime}, \pi_{1}(\Delta, \mathcal{B})$, has the same set of quasi-isometry types of one ended factors and the same number of ends.

This can be viewed as a step in Gromov's program ([G]) to classify finitely generated groups up to quasi-isometry. It effectively reduces the classification of accessible groups to the classification of one-ended groups. It would be very interesting to have a similar reduction for the classification of non-accessible groups, perhaps to some sort of quasi-conformal structure on the set of ends together with the quasi-isometry types of one-ended factors. 


\section{Basic construction}

All of our spaces are the vertex sets of connected graphs of bounded valence. We give these spaces the path metric of the graph, where every edge is considered to have length 1 . The primary motivating examples are the Cayley graphs of finitely generated groups. Different finite generating sets give different graphs, but the induced metrics on the group are bilipschitz equivalent.

If $X$ is a graph, a net in $X$ is a subset $S$ which is coarsly dense, meaning that there is an $r>0$ so that every $x$ in $X$ is within $r$ of some $s \in S$. The inclusion map $S \rightarrow X$ is a quasi-isometry when $S$ is given the induced metric. One can give $S$ a graph structure by connecting any two vertices within $2 r$ by an edge. The resulting metric is bilipschitz equivalent to the metric induced from $X$.

The free product, $G * H$, of two finitely presented groups has a nice geometric model. Bass-Serre theory gives a tree $T$ with a $G * H$ action, free on edges, with quotient an edge, and the stabilizers of the vertices the conjugates of $G$ and $H$. The model for $G * H$ is produced by "blowing up" the vertices of the tree to be copies of the Cayley graphs of $G$ and $H$, so that the $G * H$ action becomes free. The resulting space has all its vertices in these vertex subgraphs, and there is exactly one edge at every vertex connecting to another vertex space. See $[\mathrm{SW}]$ for more details and generalizations to more complicated graphs of groups.

We need to generalize this and define the free product of two spaces, $X$ and $Y$. Much of the tree of spaces structure of free products of groups makes sense for arbitrary spaces: one wants a graph with distinguished subgraphs, each isomorphic to $X$ or $Y$, which are disjoint and cover all the vertices. Every edge not in one of these subgraphs should connect a subgraph isomorphic to $X$ to one isomorphic to $Y$, and there should be precisely one such edge at every vertex. Finally, the pattern of attachments of these subgraphs should be a tree.

This description is not quite sufficient to uniquely define a graph. To construct such a tree of spaces, we start with, say, a copy of $X$ and, at every vertex of this $X$ add an edge connecting to a copy of $Y$. Immediately we run into ambiguity connecting to a copy of $Y$ at what point? This difficulty does not arise when building a free product out of Cayley graphs because they have transitive isomorphism group, which makes all possible points of attachment equivalent.

To get around this, we give our spaces $X$ and $Y$ distinguished base points, $x_{0}$ and $y_{0}$. We can then construct a canonical free product of $\left(X, x_{0}\right)$ and $\left(Y, y_{0}\right)$ as follows:

Let $\Gamma_{0}$ be the graph which is the disjoint union of $X$ and $Y$, with an edge added connecting the base points. Observe that this graph satisfies all the above conditions, except that some vertices are not incident to edges not in a subgraph, although the base point of every copy of $X$ or $Y$ is.

Given $\Gamma_{n}$ build $\Gamma_{n+1}$ as follows: For any $v$ in $\Gamma_{n}$ not incident to an edge which connects to another subgraph, add a new subgraph, isomorphic to $X$ or $Y$ as required, and an edge which connects to $v$ and to the base point of the added 
graph. One has $\Gamma_{n}$ embedded canonically as a subgraph of $\Gamma_{n+1}$, and the direct limit (union) as $n \rightarrow \infty$ is $\left(X, x_{0}\right) *\left(Y, y_{0}\right)$.

The space $\left(X, x_{0}\right) *\left(Y, y_{0}\right)$ is characterized by:

- $X * Y$ contains a disjoint collection of subgraphs, each with an isomorphism to $X$ or $Y$.

- Every vertex of $X * Y$ is contained in one of the subgraphs and is incident to exactly one edge not in that subgraph.

- Every edge not in one of the subgraphs connects a subgraph isomorphic to $X$ and a subgraph isomorphic to $Y$, and is incident to the base point of one of the components it connects. Further, there is a unique edge in $X * Y$, called the base edge, which is incident to the base points in both components it connects.

- The quotient graph in which each of the subgraphs is collapsed to a point is a tree.

As discussed above, the construction does not depend on base points for groups. For more general graphs as $X$ and $Y$ the choice of base points will affect the graph constructed by the above. The bilipschitz class of metric space is independent of these choices for a wider class of spaces.

We say that $X$ is homogeneous if $X$ has the property that for some $L$ and for any $x_{1}$ and $x_{2}$ in $X$ there is a self $L$-bilipschitz map taking $x_{1}$ to $x_{2}$. Note that this is much weaker than transitive isometry group.

Lemma 1.1. Let $X$ and $Y$ be homogeneous graphs. Let $Z$ be a graph so that for some $L>0$ :

- $Z$ contains a disjoint family of subgraphs $\left\{X_{i}\right\}$ and $\left\{Y_{i}\right\}$ whose union contains all the vertices.

- Every edge of $Z$ not in one of the subgraphs connects some $X_{i}$ to some $Y_{j}$, and there is exactly one such edge at every vertex of $Z$.

- For every $i$, there is an $L$ bilipschitz equivalence of $X_{i}$ (resp. $Y_{i}$ ) and $X$ (resp. Y).

- The quotient graph obtained from $Z$ by collapsing each of the subgraphs to a point is a tree.

There is an $M$, depending only on $L$ and the homogeneity constants of $X$ and $Y$, so that for any edge, $e$, in $Z$ connecting an $X_{i}$ and $a Y_{j}$ and any choice of base points in $X$ and $Y$, there is an $M$ bilipschitz equivalence of $Z$ to $\left(X, x_{0}\right) *\left(Y, y_{0}\right)$ taking e to the base edge.

Proof. Note that as $X$ is homogeneous there is a $K$ so that for any $X_{i}$, any $x \in X_{i}$, and any $x^{\prime} \in X$, there is a $K$ bilipschitz equivalence $X_{i} \rightarrow X$ which takes $x$ to $x^{\prime}$. The same holds for $Y_{j}$ 's mapping to $Y$.

Call the edge $e$ the base edge of $Z$. Choose bilipschitz equivalences of $X_{i} \rightarrow X$ and $Y_{j} \rightarrow Y$, as above. As $X$ and $Y$ are homogeneous we may assume that the endpoints of the base edge are $x_{0}$ and $y_{0}$. This gives a quasi-isometry between the 
union, $\Sigma_{0}$, of $X_{i}, Y_{j}$, and the base edge to the subgraph of $X * Y$ which was called $\Gamma_{0}$ in the earlier construction. This map restricts to a bijection of the edges of $\left(X, x_{0}\right) *\left(Y, y_{0}\right)$, not in $X$ or $Y$, with an endpoint in $\Gamma_{0}$ and the edges of $Z$, not in $X_{i}$ or $Y_{j}$, with an endpoint in $\Sigma_{0}$.

One now follows the construction of $X * Y$. At every stage we have a subgraph $\Sigma_{n}$ of $Z$ with a quasi-isometry to $\Gamma_{n}$ which induces a bijection of the incident edges not in the distinguished subgraphs. Let $\Sigma_{n+1}$ be the subgraph of $Z$ which contains $\Sigma_{n}$, all the $X_{i}$ and $Y_{j}$ which are adjacent to it, and the edges connecting them. Extend the map to a bilipschitz equivalence of $\Sigma_{n+1}$ and $\Gamma_{n+1}$, by choosing, for each new $X_{i}$ and $Y_{j}$ a bilipschitz equivalence with the copy of $X$ or $Y$ attached at the corresponding point of $\Gamma_{n}$ which takes the point of attachment to $x_{0}$ or $y_{0}$.

These bilipschitz equivalences give, in the limit as $n \rightarrow \infty$, the desired bilipschitz equivalence.

In particular, if $X$ and $Y$ are homogeneous then the bilipschitz type of $\left(X, x_{0}\right) *$ $\left(Y, y_{0}\right)$ does not depend on the choice of basepoints so we will usually write simply $X * Y$. This also implies that $X * Y$ is also homogeneous. All the spaces we consider are built out of coset spaces by passing to bilipschitz equivalent spaces and the free product construction, and hence are all homogeneous.

The basepoints within each copy of $X$ or $Y$ in $X * Y$ are, even for homogeneous $X$ and $Y$, a useful bookkeeping device. Choosing basepoints amounts to a choice of base edge in $X * Y$; the base points of each copy of $X$ or $Y$ is determined by being the closest point in that subgraph to the base edge.

To use Lemma 1.1, we need constructions of bilipschitz equivalences. The next two lemmas are important examples of this, and clearly demonstrate the utility of the generality of homogeneous spaces rather than simply coset spaces of groups.

Lemma 1.2. Let $X$ be infinite. Define $X^{+}$as the graph obtained from $X$ by adding a vertex $v$ which is connected by an edge to the base point of $X$. There is a bilipschitz equivalence between $X$ and $X^{+}$, hence $X * Y$ and $X^{+} * Y$ are bilipschitz equivalent for any $Y$.

Proof. As $X$ is an infinite connected graph of bounded valence there is an infinite embedded path in $X$, starting at the base point. Let $x_{0}, x_{1}, \ldots$ be such a path. Define a map from $X$ to $X^{+}$as follows:

- Send all points in $X \backslash\left\{x_{i}\right\}$ to their images under the inclusion of $X$ in $X^{+}$.

- Send $x_{0}$ to $v$.

- For $i>0$ send $x_{i}$ to $x_{i-1}$.

It is easy to verify that this is a bilipschitz equivalence.

Note that this bilipschitz equivalence of $X^{+}$and $X$ implies that if $X$ is homogeneous then so is $X^{+}$, although it will almost never have a transitive group of graph automorphisms. 
This technique of "sliding from infinity" along a path is used repeatedly in the following constructions to produce bilipschitz equivalences.

Given $X$ and $Y$, define the wedge of $X$ and $Y$ as the space obtained from the disjoint union by adding an edge connecting the base points. Notice that even if $X$ and $Y$ are homogeneous the wedge, in general, is not. Thus the choice of a base point is nontrivial. We will pick one of the endpoints of the edge joining the halves.

One important reflection of the fact that free products are tree-like is the following:

Lemma 1.3. Let $X$ and $Y$ be infinite homogeneous space, and let $Z=X * Y$. There is a bilipschitz equivalence between $Z$ and the wedge of any finite number of copies of $Z$.

Proof. By induction, we only need to prove that the wedge of two copies of $Z$ is bilipschitz equivalent to $Z$.

The base edge divides $Z$ into two infinite, connected subgraphs. Thus $Z$ is bilipschitz equivalent to the wedge of these two halves. Each half is almost $X * Y$ - it is covered by disjoint families of copies of $X$ and $Y$, connected alternately in a tree of spaces. The way in which the halves differ from $X * Y$ is that in a single subgraph (isomorphic to $X$ in one half and to $Y$ in the other) the base point is not connected to any other subgraph.

Consider the half in which the deficient subgraph is $X$. In that copy of $X$, choose a path, $p$, from the base point to infinity. Let $e_{i}$, for $i>0$, be the edges connecting $p(i)$ to points $y_{i}$ in copies of $Y$. Modify the graph structure by removing the edges $e_{i}$ and adding edges $e_{i}^{\prime}$ which connect $p(i-1)$ to $y_{i}$. This does not change the bilipschitz type, and, by Lemma 1.1, the resulting graph is bilipschitz equivalent to $X * Y=Z$.

After the analogous modification in the other half, the graph is bilipschitz equivalent to $Z$ wedge $Z$, completing the proof.

Note that this implies that for $X$ and $Y$ homogeneous, and $Z=X * Y$, that a wedge of two (and hence any finite number) of copies of $Z$ is homogeneous.

As we observe in the introduction, it is not true, even for groups, that if $G$ and $G^{\prime}$ are quasi-isometric then $G * H$ and $G^{\prime} * H$ are quasi-isometric. Thus one is motivated to ask, as in $[\mathrm{G}]$, when quasi-isometric groups are bilipschitz equivalent. In $[\mathrm{P}]$ it is shown that all non-abelian free groups are bilipschitz equivalent. In [W], where the general question of when a quasi-isometry is at bounded distance of a bilipschitz map is resolved, it is shown that any two quasi-isometric non-amenable groups are bilipschitz equivalent. No example of infinite groups which are quasiisometric but not bilipschitz equivalent is known, but $[\mathrm{BK}]$ and $[\mathrm{McM}]$ show there are graphs quasi-isometric to $\mathbb{Z}^{2}$ which are not bilipschitz equivalent to $\mathbb{Z}^{2}$.

Consider the special case of Theorem 0.1 where $A$ is a subgroup of finite index 
of $B$. There is a natural homomorphism from $A * C$ to $B * C$, but unless $C$ is trivial, it has infinite index image. There is a subgroup of $B * C$ of finite index built out of $A$ and $C$; it is isomorphic to $A * C * \ldots * C$ with $[A: B]$ copies of $C$. Thus, Theorem 0.1 implies that $A * C$ and $A * C * C$ are quasi-isometric. That special case, generalized to arbitrary spaces, is one of the key constructions in the proof of the later theorems.

Lemma 1.4. If $X$ and $Y$ are infinite homogeneous spaces then $X * Y$ and $X *$ $(Y * Y)$ are bilipschitz equivalent.

Proof. By lemma 1.2, $X * Y$ and $X^{+} * Y$ are quasi-isometric. Here we consider $X^{+}$as a space with distinguished base point the vertex $x_{0}$ which is the base point of the copy of $X$ inside it, and we let $v$ be the vertex in $X^{+} \backslash X$.

We claim $X^{+} * Y$ is bilipschitz equivalent to $X *(Y * Y)$. We prove this by modifying the graph structure of $X^{+} * Y$ without changing the bilipschitz type, and then verifying that the modified graph satisfies the properties which characterize $X *(Y * Y)$.

We first modify $X^{+} * Y$ as follows: in every copy of $X^{+}$, the vertex $v$ is connected to a base point $y_{0}$ of a copy of $Y$ and to the base point $x_{0}$ of the copy on $X$ within $X^{+}$. The vertex $x_{0}$ is also connected to a vertex $y_{1}$ in a copy of $Y$. We add an edge connecting $y_{0}$ to $y_{1}$ and delete the edge connecting $v$ to $y_{0}$. Call this modified graph $G_{0}$.

We have not changed the vertex set, and the identity map on vertices is bilipschitz between $X^{+} * Y$ and $G_{0}$. Further, $G_{0}$ is vertex-covered by subgraphs identified with $X^{+}$and $Y$. In each copy of $X^{+}$the $v$ vertex is now only connected to $x_{0}$, and not to any copies of $Y$. We wish to remove these vertices. Consider the full subgraph of $G_{0}$ which consists of all the vertices except these $v$ vertices and their incident edges. Call this subgraph $G_{1}$.

The graph $G_{1}$ is connected, and consists of copies of $X$ and copies of $Y$ and edges connecting them. By running the points in $G_{0}-G_{1}$ to infinity in the incident copies of $X$, as in the proof of lemma 1.2, one sees that $G_{1}$ and $G_{0}$ are bilipschitz equivalent.

If we delete all edges of $G_{1}$ that connect a copy of $X$ to a copy of $Y$, what remains divides into two types of components: copies of $X$ and components that are made up of copies of $Y$ 's joined by edges. We call the latter components of $Y$ 's.

We need to understand the pattern in which the copies of $Y$ are connected in a component of $Y^{\prime}$ 's. The edges connecting copies of $Y$ can be described as follows: for every copy of $X^{+}$in $X^{+} * Y$ we have, in $G_{1}$, an edge connecting the two copies of $Y$ that are connected in $X^{+} * Y$ to the base point and the "extra" point, $v$. In $X^{+} * Y$, every non base point in a copy of $Y$ connects to the base point of an $X^{+}$. Thus, every non base point in a copy of $Y$ in $G_{1}$ connects to another copy of $Y$.

We now "slide" edges in the components of $Y$ in $G_{1}$ so that every vertex 
connects to another copy of $Y$. For any component of $Y$ in $G_{1}$ whose base point does not connect to another copy of $Y$, choose a simple path, $p$, to infinity in that $Y$, starting at the base point. Modify the edge structure of $G_{1}$ as follows: for $i>0$ let $e_{i}$ be the edge at $p(i)$ connecting to another copy of $Y$, and let $y_{i}$ be the other endpoint of $e_{i}$. Remove all the $e_{i}$, and add edges $e_{i}^{\prime}$ connecting $p(i-1)$ to $y_{i}$. Let $G_{2}$ be the resulting graph.

It is clear that $G_{2}$ is bilipschitz equivalent to $G_{1}$. The graph $G_{2}$ now contains subgraphs isomorphic to $X$ and subgraphs bilipschitz equivalent to $Y * Y$ (in fact, isomorphic to $Y * Y$ aside from choices of basepoints, which does not change the bilipschitz type, by Lemma 1.1), and edges connecting them. The only difference between $G_{2}$ and $X *(Y * Y)$ is that not every point in each $Y * Y$ connects to a copy of $X$ : the base points of those components which, in $X^{+} * Y$, connect to the "extra" vertex $v$ in copies of $X^{+}$do not connect to copies of $X$ in $G_{2}$.

To fix this we again slide edges. In every copy of $Y$ in $G_{2}$ whose base point does not connect to a copy of $X$, choose a $p$ starting at the base point and running to infinity. Now let $e_{i}$, for $i>0$, be the edges connecting $p(i)$ to $x_{i}$ in copies of $X$. Let $G_{3}$ be the graph constructed from $G_{2}$ by removing the $e_{i}$ and adding $e_{i}^{\prime}$ which connects $p(i-1)$ to $x_{i}$.

The graph $G_{3}$ consists of copies of $X$ and of $Y * Y$ connected as in Lemma 1.1, and so is bilipschitz equivalent to $X *(Y * Y)$. It is, by construction, bilipschitz equivalent to $X^{+} * Y$ and therefore to $X * Y$, completing the proof.

As discussed above, the lemma is false for some cases of finite $X$ or $Y$. It is not difficult, using the above techniques, to determine the truth in that case, but the answer is somewhat complicated. For the cases arising from groups, this is analyzed at the start of the proof of Theorem 0.1 in the next section.

\section{Building quasi-isometries}

In this section we show how to use the constructions of the last section to build quasi-isometries between various graphs of groups, proving Theorems $0.1,0.2$, and 0.3 .

We start with the proof of Theorem 0.1 .

Proof. First, if $A$ (and therefore $B$ ) and $C$ are finite, one knows that $A * C$ and $B * C$ are virtually free. Under the assumptions on cardinality, they are not virtually cyclic, and hence are quasi-isometric.

Second, if $A$ and $B$ are finite, but $C$ is infinite, then $A * C$ and $B * C$ contain subgroups of finite index isomorphic to $C * \ldots * C(|A|$ factors $)$ and $C * \ldots * C(|B|$ factors) respectively. These are quasi-isometric by lemma 1.4 .

Likewise, if $A$ and $B$ are infinite, but $C$ is finite, then $A * C$ and $B * C$ contain subgroups of finite index isomorphic to $A * \ldots * A$ and $B * \ldots * B$, both with $|C|$ 
factors. Again, by lemma $1.4 A * \ldots * A$ is quasi-isometric to $A * A$ and $B * \ldots * B$ is quasi-isometric to $B * B$.

Therefore it suffices to prove the theorem when $A, B$, and $C$ infinite. We assume now that this is the case. By lemma 1.4, it suffices to prove that $A * C * C$ and $B * C * C$ are quasi-isometric. So we assume, from now on, that $C$ splits as a free product of infinite groups.

Let $f: A \rightarrow B$ be a quasi-isometry. There are nets $X$ in $A$ and $Y$ in $B$ so that $f$ induces a bilipschitz equivalence $X \rightarrow Y$. We can choose such $X$ and $Y$ to include the base points of $A$ and $B$. Let $r_{1}: A \rightarrow X$ and $r_{2}: B \rightarrow Y$ be projections onto the nets, moving points a uniformly bounded distance. Choose these projections so that only the base point of $A$ maps to the base point of $X$, and likewise only the base point of $B$ maps to the base point in $Y$.

Consider the space $A * C$. Inside each copy of $A$, for each vertex $a$, there is an edge $e$ which connects $a$ to some $c$ in a copy of $C$. Remove that edge, and replace it by an edge connecting $c$ to $r_{1}(a)$. Since the distance between $a$ and $r_{1}(a)$ is uniformly bounded, the new graph is bilipschitz equivalent to $A * C$. All the edges leaving each copy of $A$ do so at a point of $X$, hence we can replace each copy of $A$ by a copy of $X$ without changing the quasi-isometry type.

What we now have is not quite $X * C$, since each point of $X$ connects to possibly more than one copy of $C$. For each $x$ in a copy of $X$, pick one of the copies of $C$ connected to $x$, and slide all the edges connecting $x$ to other copies of $C$ to connect to the chosen copy of $C$ instead of $x$. Note that if there is more that one copy of $C$ connected to $x, x$ is not the base point of $X$, and so connects to the base point of the copies of $C$.

The resulting graph is a tree of spaces, with copies of $X$ connecting to spaces which are made of copies of $C$ attached to each other by edges joining their base points, in other words, wedges of finite number of copies of $C$. Since $C$ splits as a free product of infinite groups, Lemma 1.3 shows each of these wedges is bilipschitz equivalent to $C$. Thus $A * C$ is quasi-isometric to $X * C$ by Lemma 1.1.

By the same construction $B * C$ is quasi-isometric to $Y * C$. Since $X$ and $Y$ are bilipschitz equivalent, $X * C$ and $Y * C$ are bilipschitz equivalent, which completes the proof. We note that the proof goes through unchanged for homogeneous spaces rather than groups.

Stallings' Ends theorem says that any group with infinitely many ends splits non-trivially over a finite group, thus we want to extend Theorem 0.1 to cover such splittings. This is the content of Theorem 0.2 , which we now prove.

Proof. To start, consider a free product with amalgamation $A *_{F} B$, with $F$ a finite normal subgroup of both $A$ and $B$. In this case we have $F$ as a normal subgroup of $A *_{F} B$, with quotient $(A / F) *(B / F)$. Thus $A *_{F} B$ is quasi-isometric to $(A / F) *(B / F)$. Since $A / F$ is quasi-isometric to $A$ and $B / F$ is quasi-isometric to $B$, Theorem 0.1 proves $A * B$ and $A *_{F} B$ are quasi-isometric. 
When $F$ is not normal in $A$ or $B$, we make the same argument, but now $A / F$ and $B / F$ are spaces rather than groups. In the tree of spaces modeling $A *_{F} B$ (see $[\mathrm{SW}]$ ), one has copies of $A$ and $B$, but rather than single edges connecting copies of $A$ to $B$, one has an $F$ coset in $A$ joined to an $F$ coset in $B$ by $|F|$ edges.

Choose $X$ in $A$ so that $X$ contains one point of each coset $a F$. Give $X$ the structure of a graph by joining $x$ and $x^{\prime}$ by an edge if there is an edge joining points in the corresponding cosets. Likewise, choose $Y$ in $B$ which intersects every $F$ coset in one point. Replacing each $A$ by $X$ and $B$ by $Y$, with an edge joining $x \in X$ and $y \in Y$ if and only if the corresponding cosets are connected in $A *_{F} B$, gives a quasi-isometry between $A *_{F} B$ and $X * Y$. As $X$ is quasi-isometric to $A$ and $Y$ is quasi-isometric to $B$, it follows exactly as in the proof of Theorem 0.1 that $A * B$ is quasi-isometric to $X * Y$. Thus $A *_{F} B$ and $A * B$ are quasi-isometric.

The situation for $A *_{F}$ is similar. The model for $A *_{F}$ is a tree of spaces, each of which is copy of $A$. The edges between copies of $A$ are directed, attaching a coset of the first embedding of $F$ in $A$ in one copy of $A$ to a coset of the second embedding of $F$ in $A$ in another copy of $A$. Every point is connected to two other copies of $A$, once as an initial vertex and once as a terminal vertex.

To carry out the same argument, one needs to find a subset $X$ of $A$ which is simultaneously a set of coset representatives for both embeddings of $F$ in $A$. That such an $X$ exists is a standard application of Hall's Marriage Lemma ([GW]). Given such an $X$, the argument above.

Having proven Theorems 0.1 and 0.2 , it is straightforward to deal with an arbitrary graph of groups with finite edge groups, as such a graph of groups is simply iterated free products with finite amalgamation and HNN extensions over finite subgroups $([\mathrm{S}])$.

In order to prove Theorem 0.3 , we prove a slightly different result which clearly implies it, but is somewhat more awkward.

Theorem 2.1. Let $G$ be a graph of groups with finite edge groups. Let $S$ be the set of quasi-isometry types of vertex spaces, without repetition. Let $G^{\prime}$ be the free product of a finite set of groups with quasi-isometry types representing every type of $S$ exactly once. Let $F$ and $F^{\prime}$ be any (possibly trivial or cyclic) free groups so that $G * F$ and $G^{\prime} * F^{\prime}$ have the same number of ends, then $G * F$ and $G^{\prime} * F^{\prime}$ are quasi-isometric.

Proof. We prove this by induction on the number of edges in the graph. If there are no edges the result is essentially a tautology, given that one knows the number of ends is a quasi-isometry invariant which classifies free groups up to quasi-isometry.

As a graph of groups with $n+1$ edges can be built out of graphs with fewer edges, either by free product or HNN extension, the result follows from the following, which is immediate from Theorems 0.1 and 0.2 . 
Theorem 2.2. If $A$ is quasi-isometric to $A^{\prime}$ and $B$ is quasi-isometric to $B^{\prime}$, and $F$ a finite proper subgroup of $A$ and $B$, then $A *_{F} B$ is quasi-isometric to $A^{\prime} * B^{\prime}$ unless both products are virtually free. Likewise $A *_{F}$ is quasi-isometric to $A^{\prime} * \mathbb{Z}$ unless both are virtually free.

\section{Obstructing quasi-isometries}

In this section we prove Theorem 0.4, a partial converse to the earlier theorems. In view of Theorem 0.3 , and the fact that accessibility is a quasi-isometry invariant ([TW]), this comes down to:

Theorem 3.1. Let $G_{1}$ and $G_{2}$ be quasi-isometric groups, both of which are fundamental groups of terminal graphs of groups. If $H$ is a one-ended vertex group of $G_{1}$ then there is a one-ended vertex group of $G_{2}$ which is quasi-isometric to $H$.

Lemma 3.2. Let $G$ be the fundamental group of a terminal graph of groups, and let $H$ be a one-ended group. For any $(A, B)$ there is an $R$ so that for any $(A, B)$ quasi-isometric embedding $f$ of $H \rightarrow G$ there is a (necessarily unique) vertex space $X$ in $G$ with $H$ contained in the $R$ neighborhood of $X$.

Proof. Let $D$ be the maximal diameter of an edge space of $G$. For any such edge space $E$, the pre-image $f^{-1}(E)$ in $H$ has diameter at most $A(D+B)$. By one-endedness, there is some $D^{\prime}$ so that all but one of the components of the complement of any set $S$ of diameter at most $A(D+B)$ lie entirely within $D^{\prime}$ of $S$.

Thus, for any edge space $E$, the image of $f(H)$ is contained in the $A D^{\prime}+B+1$ neighborhood of one side of $E$. If $f(H)$ is not contained within twice this distance of any vertex, then we can orient every edge to point towards the half containing $f(H)$, and there is at least one edge pointing away from every vertex. Thus there are unbounded oriented rays. On the other hand, if $v$ is a vertex space which has nontrivial intersection with $f(H)$, then every edge more than $A D^{\prime}+B+1$ from $v$ must be oriented towards $v$, which contradicts the existence of unbounded oriented rays. Thus the hypothesis that $f(H)$ is not contained in a $2\left(A D^{\prime}+B+1\right)$ neighborhood must be false.

Theorem 3.1 now follows easily. The lemma shows $f(H)$ must be contained in a neighborhood of a vertex space $K$ of $G_{2}$. Applying the lemma to the inverse quasi-isometry, $f^{\prime}$, gives $f^{\prime}(K)$ contained in a neighborhood of some $H^{\prime}$ in $G_{1}$. Thus $f^{\prime} f(H)$ is contained in a neighborhood of $H^{\prime}$. Since $f^{\prime} f$ is within bounded distance of the identity map, the implies $H$ is contained in a neighborhood of $H^{\prime}$ which implies $H=H^{\prime}$. Further, the fact that a neighborhood of $f^{\prime} f(H)$ contains $H$ implies that a neighborhood of $f(H)$ contains $K$, so $f$ restricts to give a quasi- 
isometry between $H$ and $K$.

Theorem 0.4 reduces the large scale geometry of accessible groups to the large scale geometry of one-ended groups. It would be very interesting to understand the geometry of non-accessible groups.

Acknowledgements. The first author would like to thank Pierre de la Harpe, Thomas Delzant and Frederic Haglund for conversations related to this work. The second author would like to thank Benson Farb, Lee Mosher, and Shmuel Weinberger for their suggestions and encouragement. We would also like to thank the referee for many improvements and corrections.

\section{References}

[BK] D. Burago and B. Kleiner, Separated nets in Euclidean Space, preprint.

[D1] M. J. Dunwoody, The accessibility of finitely presented groups, Invent. Math. (1985), 449-457.

[D2] M. J. Dunwoody, An inaccessible group, The Proceedings of Geometric Group Theory 1991, G. A. Niblo and M. Roller (Eds.), 75-78, LMS Lecture Notes Series 181, Cambridge University Press, 1993.

[G] M. Gromov, Asymptotic invariants of infinite groups, in Geometric group theory, (G. Niblo and M. Roller, Eds.), LMS Lecture Notes, vol. 182, Cambridge Univ. Press, 1993.

[GW] J. Graver and M. Watkins, Combinatorics with Emphasis on the Theory of Graphs, Springer-Verlag, 1977.

[McM] C. McMullen, Lipschitz maps and nets in Euclidean space, preprint.

[P] P. Papasoglu, Homogeneous Trees are Bilipschitz Equivalent, Geometriae Dedicata 54 (1995), 301-306.

[S] J.-P. Serre, Trees, Springer-Verlag, N.Y., 1980.

[St1] J. R. Stallings, On torsion-free groups with infinitely many ends, Ann. of Math. 88 (1968), 312-334.

[St2] J. R. Stallings, Group theory and three dimensional manifolds, Yale Mathematical Monographs 4, Yale University Press, New Haven, 1971.

[SW] P. Scott and T. Wall, Topological methods in group theory, London Math. Soc. Lecture Notes Ser. 36, 137-203. Cambridge University Press, 1979.

[TW] C. Thomassen and W. Woess, Vertex transitive graphs and accessibility, J. Comb. Theory, Ser. B 58, No. 2 (1993), 248-268.

[W] K. Whyte, Amenability, Bilipschitz Equivalence, and the Von Neumann Conjecture, Duke Mathematics Journal 99 (1999), 93-112.

Panos Papasoglu

Departement de Mathématiques

Université de Paris XI (Paris-Sud)

F-91405 Orsay

France

e-mail: panos@topodyn.math.u-psud.fr
Kevin Whyte

Department of Mathematics

University of Chicago

Chicago, Il 60637

USA

e-mail: kwhyte@math.uchicago.edu

(Received: April 7, 2000; revised version: October 6, 2000) 